\title{
The Role of Cardiac Magnetic Resonance in the Evaluation of Patients Presenting with Suspected or Confirmed Acute Coronary Syndrome
}

\author{
Loren P. Budge ${ }^{1}$ and Michael Salerno ${ }^{1,2,3}$ \\ ${ }^{1}$ Cardiology Division, Department of Medicine, University of Virginia Health System, 1215 Lee Street, P.O. Box 800158, \\ Charlottesville, VA 22908, USA \\ ${ }^{2}$ Department of Radiology, University of Virginia Health System, 1215 Lee Street, P.O. Box 800158, Charlottesville, \\ VA 22908, USA \\ ${ }^{3}$ Department of Biomedical Engineering, University of Virginia Health System, 1215 Lee Street, P.O. Box 800158, \\ Charlottesville, VA 22908, USA
}

Correspondence should be addressed to Michael Salerno, ms5pc@virginia.edu

Received 19 June 2011; Accepted 18 August 2011

Academic Editor: Igor Klem

Copyright (C) 2011 L. P. Budge and M. Salerno. This is an open access article distributed under the Creative Commons Attribution License, which permits unrestricted use, distribution, and reproduction in any medium, provided the original work is properly cited.

\begin{abstract}
Cardiac magnetic resonance imaging (CMR) has an important emerging role in the evaluation and management of patients who present with symptoms concerning for acute coronary syndrome (ACS). This paper discusses the role of CMR in the emergency department setting, where CMR can aid in the early and accurate diagnosis of non-ST elevation ACS in low and intermediate risk patients. For those with confirmed myocardial infarction (MI), CMR provides comprehensive prognostic information and can readily diagnose structural complications related to MI. Furthermore, the pattern of late gadolinium enhancement (LGE) seen on CMR can help determine the etiology of cardiac injury in the subset of patients presenting with ACS who do not have obstructive coronary artery disease by angiography.
\end{abstract}

\section{Introduction}

Currently, more than a third of the 5.5 million people who present to the emergency department (ED) annually in the United States with a chief complaint of chest pain are admitted to the hospital, while only about a third of those admitted are eventually given the diagnosis of acute coronary syndrome (ACS) $[1,2]$. Thus, the emergency department physician is confronted on a daily basis with patients who have symptoms worrisome for possible ACS. Available tools in the ED, which include a detailed medical history, physical exam, ECG, and serum cardiac biomarkers, have limitations for accurately and rapidly diagnosing ACS. Cardiac troponin $\mathrm{I} / \mathrm{T}$ are quite sensitive for detecting myocardial injury, but often do not become elevated until hours after an infarction has occurred and may not be elevated in ACS without myocardial infarction. Triaging of these patients may be straightforward for high-risk patients, especially those with positive serum biomarkers (troponin I or $\mathrm{T}$ or myocardial fraction of creatine kinase) or with evidence of myocardial infarction (MI) on an electrocardiogram (ECG). However, for low- and intermediate-risk patients without immediate evidence of ischemia or MI on presentation, the decision of whether to admit can often be challenging. Furthermore, there is evidence that a small, but clinically significant number of patients are discharged from the ED with a missed diagnosis of ACS, including MI [3]. Missing the diagnosis of ACS and inappropriately discharging patients can result in adverse patient outcomes and is the leading cause of malpractice lawsuits and verdicts for physicians in the emergency room [4]. Because of the high prevalence and mortality associated with ACS, long observation times or admission to the hospital is often required. Even after patients have "ruled out" for MI, further functional or 
anatomic testing is frequently performed for risk stratification. An ideal test for ACS in the ED would be able to quickly, accurately, and noninvasively triage patients with ACS symptoms shortly after arrival to the ED. Cardiac magnetic resonance imaging $(\mathrm{CMR})$ is proving to be a powerful tool for the early identification or exclusion of ACS as the cause of a patient's chest pain syndrome. Patients with evidence of MI on presentation to the ED often undergo an early invasive strategy consisting of cardiac catheterization with potential percutaneous coronary intervention (PCI). In this setting, CMR, performed after the intervention, has been shown to provide valuable prognostic information. Patients at low risk for ACS following CMR examination can potentially be discharged, thus reducing cost from unnecessary hospital admission. Additionally, for the challenging subset of patients with evidence of MI on presentation, but who have normal coronary arteries at catheterization, CMR is uniquely able to identify the correct etiology in the majority of cases. This paper will discuss the role of CMR in the early evaluation of patients presenting to the ED with symptoms concerning for ACS, the role of CMR after MI, and the role of CMR for assessing patients presenting with ACS who are found to have nonobstructive CAD by cardiac catheterization.

\section{CMR Techniques for Evaluating ACS}

CMR is capable of performing a rapid and comprehensive evaluation of cardiac anatomy, function, and myocardial perfusion at rest and/or during stress. CMR can accurately identify the presence of infarction, myocardial edema, microvascular obstruction, and intramyocardial hemorrhage. The sequence of a typical CMR exam for ACS usually begins with anatomic images of the chest, which can often provide clues about other pathologies that may be causing the patient's chest pain. Cine steady-state free precession imaging provides an assessment of myocardial and valvular function and enables accurate assessment of left ventricular ejection fraction (LVEF) and ventricular volumes. CMR can assess myocardial edema and inflammation using $T_{2}$ weighted imaging due to the increased water content of the myocardium. Edematous tissues with higher water content have longer $T_{2}$ relaxation times, leading to a brighter signal on $T_{2}$-weighted images. First-pass myocardial perfusion is assessed using saturation-recovery gradient echo techniques during intravenous injection of gadolinium contrast agents. This can be performed during pharmacologic stress using adenosine or dobutamine infusion providing an assessment of myocardial ischemia. Myocardial scar is assessed by late gadolinium-enhanced (LGE) imaging, the pattern of which can help differentiate infarction from nonischemic processes. Regions with myocardial scar have a higher concentration of contrast agent and, thus, appear bright in these images. Conversely, areas of microvascular obstruction, thrombus, and myocardial hemorrhage appear dark on LGE imaging. A complete CMR protocol can be performed in less than 45 minutes providing a rapid comprehensive assessment of the heart.

\section{Deciding Whether to Admit}

CMR has been shown to improve both the diagnostic accuracy and time to diagnosis of ACS in the emergency department setting. This was demonstrated in a study of 161 patients presenting to the ED within 12 hours of onset of symptoms concerning for ACS, but without ST elevation on ECG [5]. The CMR exam in this study included cine MRI for LV function, resting first-pass perfusion, and LGE. Total CMR time was 38 minutes, and patients were gone from the ED for less than 1 hour. The sensitivity and specificity for diagnosis of ACS by CMR was $84 \%$ and $85 \%$, respectively, and added independent diagnostic value to clinical parameters [5]. The main limitation of CMR in this study was its inability to differentiate acute from chronic MI, a problem which was addressed in a subsequent study by Cury et al., who added $T_{2}$-weighted imaging and LV wall thickness quantification to distinguish acute events [6]. $T_{2}$ weighted imaging enables visualization of edema associated with acute, but not chronic, myocardial infarction [7]. In this study, 62 patients with chest pain, normal ECG, and negative initial cardiac biomarkers, who were being admitted to the hospital to rule out $\mathrm{MI}$, received CMR with an average scan time of 32 minutes. CMR with $T_{2}$-weighted imaging accurately identified all acute MI cases, often before cardiac biomarkers became elevated. Compared with conventional CMR, the addition of $T_{2}$-weighted edema imaging and wall thickness quantification increased specificity from $84 \%$ to $96 \%$, positive predictive value from $55 \%$ to $85 \%$, and overall accuracy from $84 \%$ to $93 \%$; respectively, while sensitivity remained unchanged at $85 \%$ [6].

\section{CMR without Initial Evidence of MI}

A large proportion of low- and intermediate-risk patients presenting to the ED with symptoms concerning for ACS without evidence of MI undergo additional functional or anatomic imaging on either an inpatient or outpatient basis. CMR has been shown to compare favorably to other noninvasive cardiac imaging modalities for diagnosing ischemia. In a study by Nagel et al., 208 patients with suspected CAD underwent both dobutamine stress echo and dobutamine stress CMR prior to cardiac catheterization [8]. With CMR, sensitivity and specificity for detecting a 50\% coronary stenosis was increased from $74.3 \%$ to $86.2 \%$ and from $69.8 \%$ to $85.7 \%$, respectively, compared with echocardiography. In a study of 163 patients with poor acoustic windows preventing adequate imaging by dobutamine stress echo, Hundley et al. successfully performed dobutamine stress MRI in 153 of the patients [9]. The sensitivity and specificity for detecting a $50 \%$ coronary stenosis were $83 \%$ and $83 \%$, respectively, in the 41 patients undergoing coronary angiography within 6 months of their stress test. In the 103 patients with negative stress tests, the event free survival at a median followup of 228 days was 97\% [9]. A meta-analysis of dobutamine stress CMR including 14 studies (754 patients) with a high prevalence of coronary disease (prevalence: $70.5 \%$ ) demonstrated a sensitivity of $83 \%$ and specificity of $86 \%$ [10]. Adenosine stress cardiac MRI has also been shown 


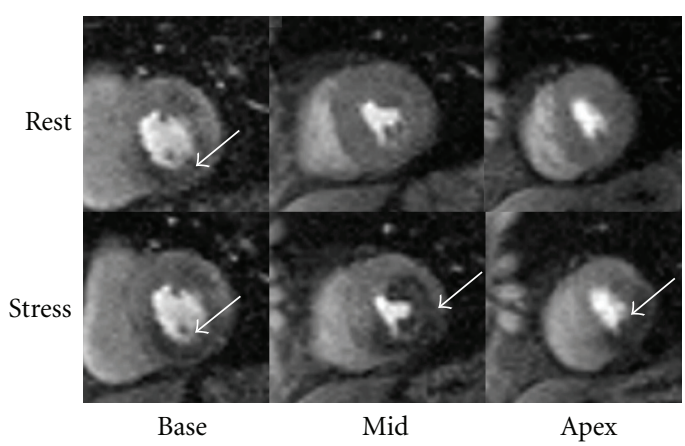

FIgURE 1: CMR with pharmacologic stress. An example of an adenosine stress CMR in a diabetic patient presenting with new onset chest pain. Resting first-pass perfusion images (top panels) are shown above the corresponding stress perfusion images (bottom panels) in the basal, midventricular, and apical short-axis views. There are inferior and anterior resting perfusion defects in the basal short-axis slice, but with stress this patient develops a severe inferior perfusion defect extending to the lateral wall and septum in the midventricle and apex. On cardiac catheterization (not shown), this patient had multivessel CAD.

to be both sensitive and specific for detection of CAD. A meta-analysis including 1658 patients with intermediate likelihood of disease undergoing adenosine stress perfusion MRI demonstrated a sensitivity of $90 \%$ and specificity of $81 \%$. The negative likelihood ratio of adenosine stress in this study was 0.14 [11]. A recent multicenter trial of 234 patients who were studied with both single photon emission computed tomography (SPECT) and CMR perfusion imaging demonstrated superior diagnostic utility for CMR perfusion at the ideal contrast dose as compared to SPECT [12]. In lower-risk populations presenting to the ED with chest pain, negative ECG, and negative serial cardiac biomarkers, CMR was performed in two separate studies evaluating a total of 192 patients who received adenosine stress and rest CMR, LV function assessment, and LGE $[13,14]$. None of the patients with a normal CMR in either of these studies had any clinical events at 9-month followup. This was corroborated in a similar study of 135 low-risk patients presenting to the ED with chest pain who received adenosine stress CMR [15]. At 1 year followup, no patients with a normal stress CMR were found to have a significant coronary stenosis or cardiac event, yielding a sensitivity and specificity of $100 \%$ and $93 \%$, respectively. Figure 1 shows an example of an adenosine stress CMR study in a diabetic patient presenting with new onset chest pain. Stress imaging demonstrates a large area of ischemia. This patient was found to have multivessel CAD.

The costeffectiveness of using stress CMR to evaluate intermediate- and high-risk patients in the ED setting was evaluated in a study of 110 patients with chest pain, negative ECG, and negative initial biomarkers, being admitted to the hospital to rule out MI [16]. These patients were randomized to obtain adenosine stress CMR while in the ED or usual inpatient care. All health care costs relating to their hospitalization and cardiac care over 30 days were included. There was no difference in 30-day outcomes between the groups. A diagnostic protocol which included stress CMR was found to reduce inpatient admissions and produced a cost savings of over $20 \%$ in this study [16]. Further cost-effectiveness studies are still needed to address the financial implications of utilizing stress CMR in lower-risk populations.

\section{CMR following Myocardial Infarction}

For those patients who present early after symptom onset with ST elevation MI (STEMI) or without ST elevation, but with elevated cardiac biomarkers (non ST elevation MI, or NSTEMI), an early invasive strategy with cardiac catheterization is generally recommended [17]. In these situations, it is usually not helpful to perform noninvasive cardiac testing prior to catheterization, because such tests could delay treatment and would not be expected to affect short-term patient management. CMR can be performed safely in MI patients, even immediately after percutaneous intervention $[18,19]$, add important prognostic information, diagnose post-MI complications, and aid in deciphering the etiology of cardiac injury in patients who present with STEMI, but who are found to have normal coronary arteries at catheterization. Figure 2 shows images from a patient who had a CMR study shortly after presenting with acute chest pain. In this case, edema imaging enabled appropriate determination of the LAD as the culprit vessel, even though there was no LGE in this region. The LGE in the lateral wall was from a prior myocardial infarction.

5.1. Prognosis. Left ventricular function has long been known to predict outcomes after MI. Impaired LV systolic function and an elevated LV end-systolic volume (ESV) are both powerful independent predictors of increased mortality [20-24]. CMR has become the gold standard for accurate quantification of left ventricular function and volumes. In contemporary CMR studies of post-MI patients, LVEF and LVESV are consistently predictive of outcomes; however, multiple studies have shown that other CMR-based parameters of infarction, such as infarct size, microvascular obstruction (MO), and myocardial salvage are more powerful predictors of outcomes than simple LV function or volume assessments [25-27].

5.2. Infarct Size. CMR-based assessment of infarct size using late gadolinium enhancement (LGE) is well validated and has been shown to be highly predictive of cardiac events at followup. Yokota et al. evaluated 86 patients with prior MI by CMR and found that infarct size by LGE was a better predictor of cardiac events than LVEF, LVESV or LVEDV, at 20 month follow up [27]. Cheong et al. followed a cohort of 857 patients with and without coronary artery disease for a median of 4.4 years and demonstrated that LGE, LVEF and heart failure symptoms were independent predictors of mortality [28]. Interestingly, patients with LGE and an ejection fraction more than $50 \%$ had the same outcomes as those with an LVEF less than 50\% without LGE [28]. A study by Larose et al. investigated 103 acute ST elevation MI (STEMI) patients by CMR within 12 hours of 


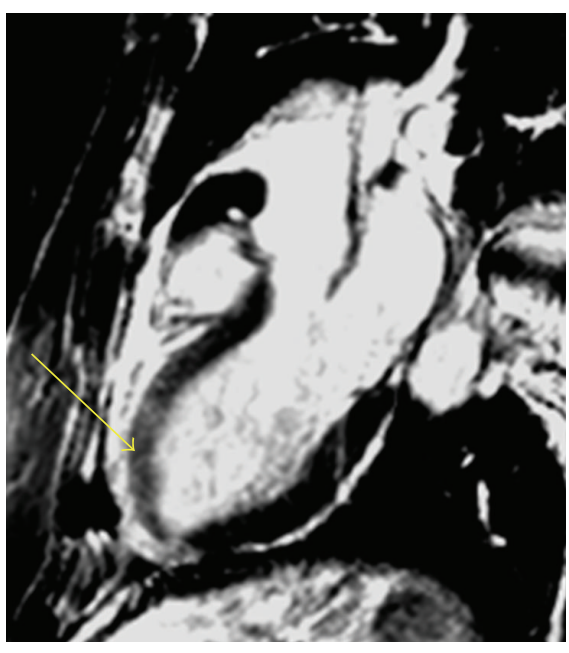

(a)

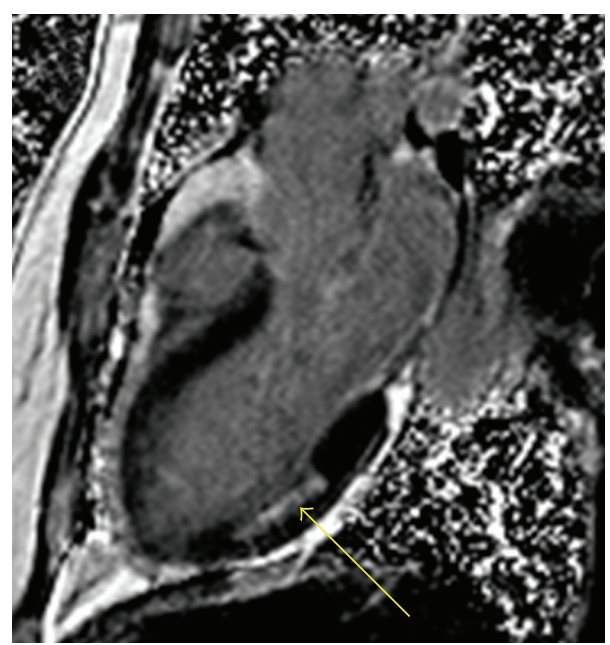

(b)

FIGURE 2: Determing acute from chronic infarcts. This patient had a CMR shortly after presenting with acute-onset chest pain. $T_{2}$-weighted imaging (a) reveals a bright signal in the mid-anteroseptum and apex, signifying edema, a marker of acute myocardial injury. Late gadolinium enhanced (LGE) imaging (b) shows an infarct in the mid-inferolateral wall, but minimal enhancement in the anteroseptum or apex. This patient had a severely stenosed mid-LAD as well as an occluded obtuse marginal branch of the circumflex coronary artery. CMR in this case was able to determine the culprit vessel.

PCI, after which they received a 6-month CMR and were followed for clinical events for an average of 2.4 years [29]. It was demonstrated that LGE measured immediately after revascularization strongly and independently predicted 6month LVEF and long-term major cardiac events better than LVEF or clinical parameters. This was corroborated in a study of 122 STEMI patients who received CMR after revascularization and were followed for 2 years. Infarct size was again a stronger indicator of worsened LV function and clinical outcomes at followup than were baseline measurements of LV systolic performance [30].

5.3. Microvascular Obstruction. Microvascular obstruction (MO) refers to severe capillary and endothelial damage induced by prolonged ischemia, which prevents blood flow into the infarcted core after reperfusion. The extent of MO usually peaks between 2 hours to 2 days after reperfusion occurs [31-33], after which it tends to be stable in size until at least day 9 [34]. Thereafter areas of MO involute and regress and are rarely seen on followup CMR beyond 1 to 2 months after MI.

CMR has become the gold standard for assessment of MO, due to its excellent spatial resolution and tissue characterization ability. MO can be assessed by CMR using two separate techniques. Early $\mathrm{MO}$ is visualized early after contrast injection using first-pass perfusion. Typical protocols using this method define MO as hypoenhancement of the myocardium persisting longer than 1 to 2 minutes after initial contrast injection. The second technique collects late LGE images 10 to 15 minutes after contrast injection, and MO appears as an area or areas of hypoenhancement encompassed within a core of enhanced infarcted myocardium, often extending from the subendocardium. This latter method in the literature is sometimes termed persistent or late MO. Both methods of MO assessment have been validated, and there is not yet a clear consensus as to which method should be the preferred technique. Early MO has been shown to be predictive of long-term infarct size, infarct transmurality, LV function, and LV remodeling and is also a powerful independent long-term prognostic indicator of hard cardiac events even after controlling for infarct size $[26,35]$; however, timing of the CMR examination after MI is important when assessing $\mathrm{MO}$ using first-pass perfusion. Early MO was more often seen when CMR was performed 2 days after MI than on days 7 or 9 but was not predictive of long-term functional or clinical outcomes until the later time points $[34,35]$. Late MO has also been shown to lend additional prognostic information beyond that of infarct size or transmurality. In a study of $110 \mathrm{MI}$ patients, Hombach et al. found that late MO independently portended an increase in cardiac events at 8-month followup and gave additional prognostic information compared to infarct size alone [36]. This was corroborated in a CMR study of 67 STEMI patients followed for 14 months, in which late MO was a better predictor of adverse cardiac events than infarct size or baseline ejection fraction [37]. Data comparing early and late MO are sparse, but, in a study by Nijveldt et al., 63 acute MI patients who received PCI and optimal medical management were followed by CMR with assessment of early, mid-, and late MO 4 to 7 days after MI and followup CMR at 4 months. In this study, late $\mathrm{MO}$ was a better predictor of follow up LV ejection fraction, LV end-diastolic volume, and LV endsystolic volume than early $\mathrm{MO}$ [38].

5.4. Area at Risk/Myocardial Salvage. The region of ischemic myocardium within the perfusion bed of an occluded coronary artery is the "area at risk" of infarction and is potentially salvageable with appropriate and timely intervention. 
The area at risk, as measured by $T_{2}$-weighted CMR, correlates very well to the area at risk determined histologically with fluorescein staining [39]. Similarly, $T_{2}$-weighted in vivo CMR measurement of the area at risk 2 days following MI showed excellent correlation to the area at risk determined by fluorescent microspheres at the time of coronary occlusion [40].

Accurate assessment of both infarct size and the area at risk allows noninvasive measurement of a potentially clinically important parameter: the amount of myocardial salvage. The myocardial salvage index (MSI) is a measure of the myocardium that has been spared infarction, presumably due to a given procedure or treatment, and has important prognostic significance. CMR trials of STEMI patients undergoing PCI have repeatedly shown that an increase in the time from symptom onset to PCI leads to a significant incremental increase in infarct size and microvascular obstruction, with a corresponding decrease in myocardial salvage, while the extent of myocardial edema, or area at risk, remains unchanged [25, 41]. A trial of 208 STEMI patients who underwent PCI within 12 hours of symptom onset demonstrated that patients with an MSI above the study median experienced significantly fewer death, reinfarction, or heart failure events at 6 months when compared with those with an MSI below the median $(2.9 \%$ versus $22.1 \%, P<0.001$ ) [25]. MSI was found to be a better predictor of outcome on multivariate regression than infarct size, MO, LVEF, TIMI flow-grade after PCI or ST segment resolution. Because of the unique ability of $T_{2}$-weighted CMR to detect myocardial salvage and predict adverse events, it may become an extremely valuable surrogate measure of outcome in studies of reperfusion therapy.

5.5. Right Ventricular Infarction. Another prognostic indicator after MI is the presence of right ventricular (RV) infarction. RV infarction has been associated with an increased risk of cardiogenic shock, atrioventricular conduction block, and rupture of the interventricular septum [42]. In-hospital mortality after RV infarction has been reported to be as high as $50 \%$ but, among survivors to hospital discharge, does not seem to predict long-term outcomes [43]. CMR has been demonstrated to be much more sensitive for detecting small or medium RV infarcts than physical exam, ECG with rightsided leads, or echocardiography [44, 45]. RV infarction, as seen by LGE of the RV after MI, was shown to be predictive of RV dilation at 6-month followup [46]. Furthermore, a recent CMR study of $50 \mathrm{MI}$ patients undergoing successful PCI and followed for an average of 32 months reported RV involvement by LGE in $47 \%$ and $65 \%$ of inferior and anterior MIs; respectively, and found that RV infarction was a significant independent predictor of cardiac events (odds ratio: 15.8; 95\% CI: 4-63), even after controlling for LV infarct size and location [45].

5.6. Postinfarct Complications. Due to CMR's unique tissue characterization abilities and spatial resolution, it is an ideal imaging modality for assessing complications arising after MI. CMR is widely considered the gold standard for analyzing both regional and global ventricular function after MI [47] and can comprehensively evaluate both systolic and diastolic function [48]. CMR can also readily detect ventricular wall pathology such as ventricular septal defects or free-wall aneurysms and can differentiate aneurysms from pseudoaneurysms, with its accompanying treatment implications. Visualization of LGE within the papillary muscles is indicative of infarction and has been reported in post-MI CMR studies to occur in 26 to $53 \%$ of patients $[36,49]$. In a study of 60 patients with old MI, bilateral papillary muscle infarction was seen by LGE in $17 \%$ of cases and was associated with a higher incidence of severe mitral regurgitation and LV remodeling than in those with unilateral or no papillary muscle infarction [49]. Inflammatory pericarditis is visualized by LGE of the pericardium with high sensitivity and specificity after MI [50] and has been reported to occur in as many as $40 \%$ of STEMI patients [36]. CMR can also be beneficial in the evaluation of intraventricular thrombi. The presence of intraventricular thrombi has been reported in 7 to $29 \%$ of patients with decreased ejection fraction, depending on the population studied $[51,52]$, and is associated with a higher risk of thromboembolic events, including stroke $[51,53]$. The most prominent risk factors for the development of LV thrombus include anterior infarction, infarct size, LV aneurysm, and decreased ejection fraction $[52,54]$. A CMR protocol which includes LGE assessment has been shown to more accurately diagnose thrombi within the ventricle than transthoracic echocardiography (TTE) or transesophageal echocardiography (TEE). In a multimodality imaging, study of 361 patients with ischemic cardiomyopathy undergoing cardiac surgery, the presence of LV thrombus by CMR, TTE, and TEE were compared to pathology and were found to have sensitivities and specificities of $88 \%$ and $99 \%$ for CMR, $23 \%$ and $96 \%$ for TTE, and $40 \%$ and $96 \%$ for TEE, respectively [51]. While contrast echocardiography nearly doubles the sensitivity of noncontrast echo, mural, and small apical LV thrombi are still frequently missed [55]. Recently, the use of prolonged inversion times $(600 \mathrm{~ms})$ during LGE imaging has been shown to increase the sensitivity of LGE for detecting thrombus even further [52]. Figure 3 shows images from a patient who presented late after an STEMI demonstrating a large area of LGE and the presence of an apical thrombus.

\section{Myocardial Injury with Normal Coronary Arteries}

An estimated $9-14 \%$ of patients who are diagnosed with STEMI will be found to have normal coronary arteries at cardiac catheterization [56]. These patients are at similar risk for mortality as those with CAD and often have an underlying pathology which can be difficult to diagnose. CMR is uniquely able to shed light on the underlying pathology in the majority of patients with MI with normal coronary arteries. The pattern of late gadolinium enhancement and myocardial edema in these patients is often helpful in differentiating the cause of injury. Ischemic necrosis begins in the subendocardium before extending to the epicardium; thus, 


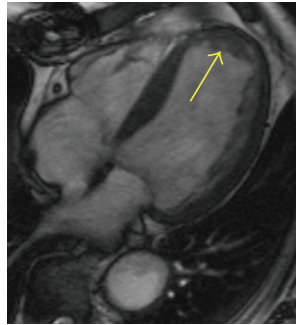

(a)

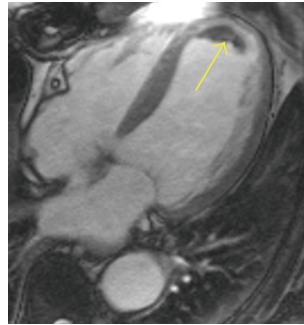

(b)

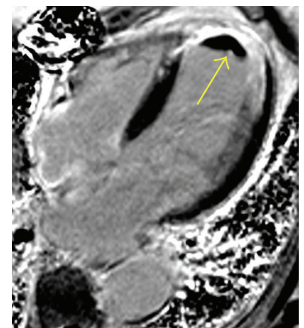

(c)

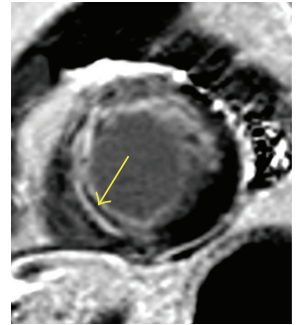

(d)

FIGURE 3: CMR imaging after acute MI. The tissue characterization abilities of CMR are demonstrated in these frames, all of which were taken from a patient who presented late after an ST elevation MI. A 4-chamber cine steady-state free precession (SSFP) image is shown in (a). Prior to contrast, it is difficult to discern whether there is thrombus in the apex. A postcontrast 4-chamber SSFP image (b) provides greater contrast between the slightly enhancing myocardium and nonenhancing thrombus at the apex. This difference is even more clear with late gadolinium-enhanced (LGE) imaging (c)-(d), which clearly demonstrates the black thrombus at the apex adjacent to the transmural apical infarct seen in white. An apical short axis view (d) reveals an extensive infarct with an area of microvascular obstruction visualized in the septum (arrow).

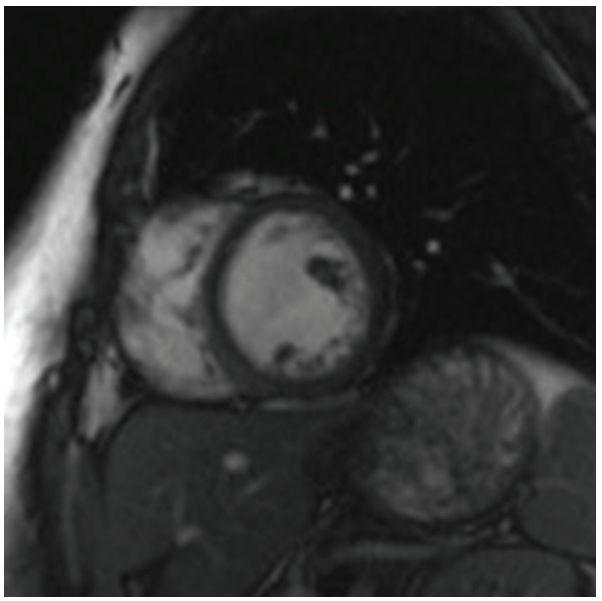

(a)

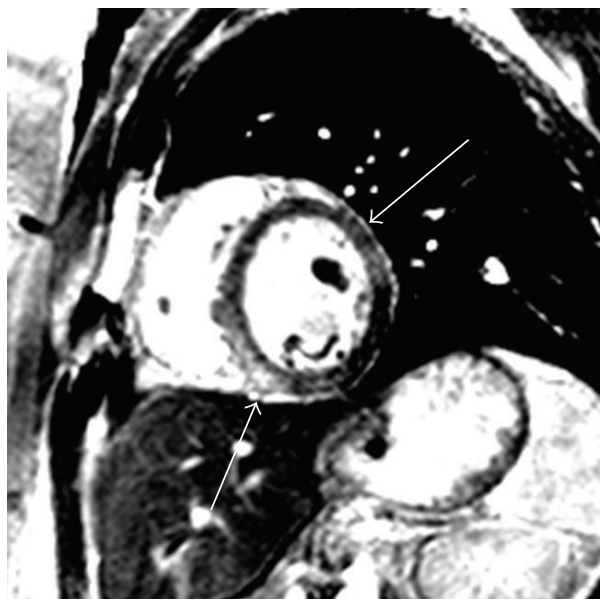

(c)

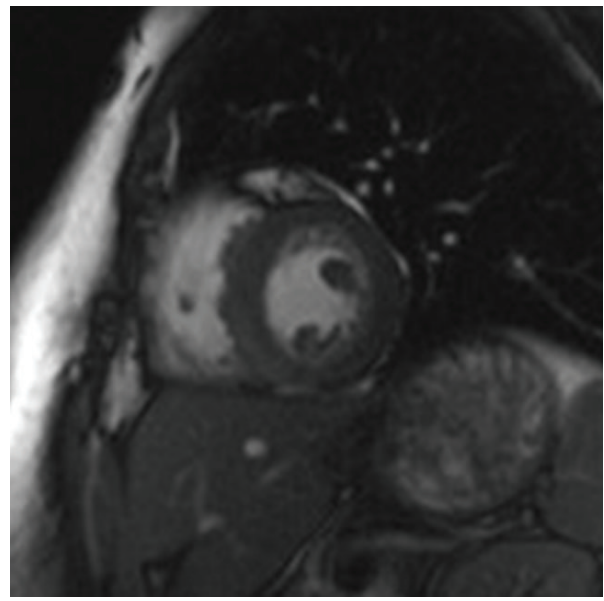

(b)

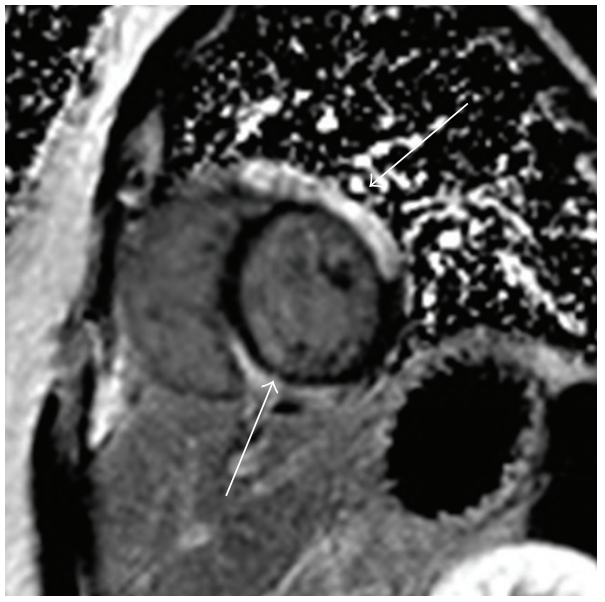

(d)

FIGURE 4: Myocardial infarction with normal coronary arteries. Images from a patient presenting with chest pain, elevated troponin but normal coronary arteries. Cine-SSFP images at (a) diastole and (b) systole demonstrate a wall motion abnormality in the inferoseptum and anterolateral walls. $T_{2}$ weighted imaging (c) shows focal areas of edema in these regions. (d) LGE imaging demonstrates epicardial-delayed enhancement. These findings are consistent with the diagnosis of myocarditis. 
sparing of the subendocardium in infarction is extremely rare. Conversely, subendocardial sparing is common in many nonischemic cardiomyopathies. Although myocarditis can present with varied LGE patterns, the classical appearance consists of a midwall or epicardial stripe of enhancement, the location of which can be indicative of the viral etiology [57, 58]. Figure 4 shows CMR images from a patient presenting with acute chest pain and a troponin elevation. Edema imaging indicated acute inflammation in the inferoseptum and anterolateral walls. LGE imaging shows epicardial scarring associated with myocarditis. Tako-Tsubo's (stress-induced) cardiomyopathy, on the other hand, does not lead to scar formation or LGE. In a study of 1345 patients with diagnosed STEMI, 127 (9.5\%) were found on angiography to have no coronary artery disease [59]. CMR in these patients had a $75 \%$ diagnostic yield and differentiated $31 \%$ as myocarditis, $31 \%$ as Tako-Tsubo cardiomyopathy, and $29 \%$ as STEMI without an angiographic lesion. This was corroborated in study of 60 patients with troponin-positive chest pain, but unobstructed coronary arteries, which also found that CMR was able to provide a specific diagnosis in the majority of patients, diagnosing $50 \%$ of these cases as myocarditis and $11.6 \%$ as MI [60]. Myocardial edema, as seen with $T_{2}$ weighted CMR, can help differentiate acute from chronic episodes of both ischemic and nonischemic events, and has been found to be present in acute episodes of TakoTsubo's cardiomyopathy [61, 62], myocarditis [63], cardiac sarcoid [64], pulmonary hypertension [65], acute transplant rejection [66], and recent cardiac surgery.

\section{Comparative Effectiveness of CMR in ACS}

In the current era of cost awareness, there is an appropriate focus on the ability of an imaging modality to improve cost and real patient outcomes when compared with other available diagnostic strategies. As already discussed, there is a relatively large body of evidence demonstrating that CMR offers excellent diagnostic and prognostic data in ACS patients and that it compares favourably to other imaging modalities. It has also been found to be cost effective in a selected population of patients presenting to the ED with suspected ACS [16]. CMR can play a key role in appropriately determining which patients with chest pain need to be admitted for further management and which patients can be safely discharged to home; this could reduce costs related to unnecessary inpatient care. There is still a great need, however, for further studies to clarify CMR's ability to directly change management, improve meaningful patient outcomes, and reduce overall costs of ACS care in the ED.

\section{Conclusions}

CMR has emerged as a robust diagnostic imaging modality capable of providing a comprehensive cardiac evaluation for patients with ACS. CMR is widely regarded as the reference standard for assessment of cardiac structure and function. In patients with acute myocardial infarction, CMR provides powerful diagnostic and prognostic information, allowing evaluation of cardiac function, infarct size and location, infarct complications, the extent of edema or area at risk, the amount of myocardial salvage, and the status of the microvasculature-all in a single scan. CMR has been shown to be safe, even immediately after PCI in STEMI patients $[18,19]$, and a comprehensive exam can typically be performed in less than 45 minutes without exposing the patient to radiation. For those with normal coronary arteries in the setting of MI, CMR provides clues to the underlying diagnosis in the majority of cases. For patients presenting to the emergency department with symptoms and risk factors concerning for ACS, but without evidence of MI, a comprehensive CMR study can also include stress perfusion imaging, allowing accurate functional assessment of the coronary circulation, which has been validated in both low and high risk study populations. $T_{2}$-weighted imaging of edema and late gadolinium enhancement have the ability to detect infarction and severe ischemia early in the course of MI, even before cardiac biomarkers become elevated, and can differentiate acute from chronic infarction. Importantly, adding CMR to the emergency physician's tool belt has been shown to improve diagnostic accuracy compared to current standards of care and to be cost effective in certain populations. Taken together, CMR provides a wealth of meaningful diagnostic and prognostic data for a wide range of patient populations with suspected or confirmed acute coronary syndrome.

\section{Abbreviations}

ACS: $\quad$ Acute coronary syndrome

CAD: Coronary artery disease

CMR: Cardiac magnetic resonance imaging

ECG: Electrocardiogram

ED: $\quad$ Emergency Department

HF: Heart failure

LGE: Late gadolinium enhancement

LV: $\quad$ Left ventricle

LVEF: Left ventricular ejection fraction

MI: $\quad$ Myocardial infarction

NSTEMI: Non-ST elevation myocardial infarction

PCI: Percutaneous coronary intervention

RV: $\quad$ Right ventricle

STEMI: ST elevation myocardial infarction

UA: Unstable angina.

\section{Disclosure}

Dr. Salerno receives research support from Siemens Healthcare.

\section{Funding}

The authors received the funds NIH 5T32EB003841, AHA 10SDG2650038. 


\section{References}

[1] F. A. Bhuiya, S. R. Pitts, and L. F. McCaig, "Emergency department visits for chest pain and abdominal pain: United States 1999-2008," NCHS Data Brief, vol. 43, pp. 1-8, 2010.

[2] J. H. Pope, R. Ruthazer, J. R. Beshansky, J. L. Griffith, and H. P. Selker, "Clinical features of emergency department patients presenting with symptoms suggestive of acute cardiac ischemia: a multicenter study," Journal of Thrombosis and Thrombolysis, vol. 6, no. 1, pp. 63-74, 1998.

[3] J. H. Pope, T. P. Aufderheide, R. Ruthazer et al., "Missed diagnoses of acute cardiac ischemia in the emergency department," The New England Journal of Medicine, vol. 342, no. 16, pp. 1163-1170, 2000.

[4] R. A. Rusnak, T. O. Stair, K. Hansen, and J. S. Fastow, "Litigation against the emergency physician: common features in cases of missed myocardial infarction," Annals of Emergency Medicine, vol. 18, no. 10, pp. 1029-1034, 1989.

[5] R. Y. Kwong, A. E. Schussheim, S. Rekhraj et al., "Detecting acute coronary syndrome in the emergency department with cardiac magnetic resonance imaging," Circulation, vol. 107, no. 4, pp. 531-537, 2003.

[6] R. C. Cury, K. Shash, J. T. Nagurney et al., "Cardiac magnetic resonance with T2-weighted imaging improves detection of patients with acute coronary syndrome in the emergency department," Circulation, vol. 118, no. 8, pp. 837-844, 2008.

[7] H. Abdel-Aty, M. Cocker, C. Meek, J. V. Tyberg, and M. G. Friedrich, "Edema as a very early marker for acute myocardial ischemia a cardiovascular magnetic resonance study," Journal of the American College of Cardiology, vol. 53, no. 14, pp. 11941201, 2009.

[8] E. Nagel, H. B. Lehmkuhl, W. Bocksch et al., "Noninvasive diagnosis of ischemia-induced wall motion abnormalities with the use of high-dose dobutamine stress MRI: comparison with dobutamine stress echocardiography," Circulation, vol. 99, no. 6, pp. 763-770, 1999.

[9] W. G. Hundley, C. A. Hamilton, M. S. Thomas et al., "Utility of fast cine magnetic resonance imaging and display for the detection of myocardial ischemia in patients not well suited for second harmonic stress echocardiography," Circulation, vol. 100, no. 16, pp. 1697-1702, 1999.

[10] K. R. Nandalur, B. A. Dwamena, A. F. Choudhri, M. R. Nandalur, and R. C. Carlos, "Diagnostic performance of stress cardiac magnetic resonance imaging in the detection of coronary artery disease: a meta-analysis," Journal of the American College of Cardiology, vol. 50, no. 14, pp. 1343-1353, 2007.

[11] M. Hamon, G. Fau, G. Née, J. Ehtisham, R. Morello, and M. Hamon, "Meta-analysis of the diagnostic performance of stress perfusion cardiovascular magnetic resonance for detection of coronary artery disease," Journal of Cardiovascular Magnetic Resonance, vol. 12, no. 1, article 29, 2010.

[12] J. Schwitter, C. M. Wacker, A. C. van Rossum et al., "MRIMPACT: comparison of perfusion-cardiac magnetic resonance with single-photon emission computed tomography for the detection of coronary artery disease in a multicentre, multivendor, randomized trial," European Heart Journal, vol. 29, no. 4, pp. 480-489, 2008.

[13] S. Lerakis, D. S. McLean, A. V. Anadiotis et al., "Prognostic value of adenosine stress cardiovascular magnetic resonance in patients with low-risk chest pain," Journal of Cardiovascular Magnetic Resonance, vol. 11, no. 1, article 37, 2009.

[14] G. Hartlage, M. Janik, A. Anadiotis et al., "Prognostic value of adenosine stress cardiovascular magnetic resonance and dobutamine stress echocardiography in patients with low-risk chest pain," International Journal of Cardiovascular Imaging, 2011.

[15] W. P. Ingkanisorn, R. Y. Kwong, N. S. Bohme et al., "Prognosis of negative adenosine stress magnetic resonance in patients presenting to an emergency department with chest pain," Journal of the American College of Cardiology, vol. 47, no. 7, pp. 1427-1432, 2006.

[16] C. D. Miller, W. Hwang, J. W. Hoekstra et al., "Stress cardiac magnetic resonance imaging with observation unit care reduces cost for patients with emergent chest pain: a randomized trial," Annals of Emergency Medicine, vol. 56, no. 3, pp. 209-219 e202, 2010.

[17] J. L. Anderson, C. D. Adams, E. M. Antman et al., "ACC/AHA 2007 guidelines for the management of patients with unstable angina/non-ST-elevation myocardial infarction: a report of the American College of Cardiology/American Heart Association Task Force on Practice Guidelines (writing committee to revise the 2002 guidelines for the management of patients with unstable angina/non-ST-elevation myocardial infarction) developed in collaboration with the American College of Emergency Physicians, the Society for Cardiovascular Angiography and Interventions, and the Society of Thoracic Surgeons endorsed by the American Association of Cardiovascular and Pulmonary Rehabilitation and the Society for Academic Emergency Medicine," Journal of the American College of Cardiology, vol. 50, no. 7, pp. e1-e157, 2007.

[18] C. M. Kramer, W. J. Rogers, and D. L. Pakstis, "Absence of adverse outcomes after magnetic resonance imaging early after stent placement for acute myocardial infarction: a preliminary study," Journal of Cardiovascular Magnetic Resonance, vol. 2, no. 4, pp. 257-261, 2000.

[19] E. Larose, J. Cote, J. Rodes-Cabau et al., "Contrast-enhanced cardiovascular magnetic resonance in the hyperacute phase of ST-elevation myocardial infarction," International Journal of Cardiovascular Imaging, vol. 25, no. 5, pp. 519-527, 2009.

[20] A. J. Moss, "Risk stratification and survival after myocardialinfarction," The New England Journal of Medicine, vol. 309, no. 6, pp. 331-336, 1983.

[21] M. B. Mock, I. Ringqvist, L. D. Fisher et al., "Survival of medically treated patients in the coronary artery surgery study (CASS) registry," Circulation, vol. 66, no. 3, pp. 562-568, 1982.

[22] A. Volpi, C. DeVita, M. G. Franzosi et al., "Determinants of 6-month mortality in survivors of myocardial infarction after thrombolysis: results of the GISSI-2 data base," Circulation, vol. 88, no. 2, pp. 416-429, 1993.

[23] B. L. Zaret, T. F. J. Wackers, M. L. Terrin et al., "Value of radionuclide rest and exercise left ventricular ejection fraction in assessing survival of patients after thrombolytic therapy for acute myocardial infarction: results of Thrombolysis in Myocardial Infarction (TIMI) phase II study," Journal of the American College of Cardiology, vol. 26, no. 1, pp. 73-79, 1995.

[24] H. D. White, R. M. Norris, and M. A. Brown, "Left ventricular end-systolic volume as the major determinant of survival after recovery from myocardial infarction," Circulation, vol. 76, no. 1, pp. 44-51, 1987.

[25] I. Eitel, S. Desch, G. Fuernau et al., "Prognostic significance and determinants of myocardial salvage assessed by cardiovascular magnetic resonance in acute reperfused myocardial infarction," Journal of the American College of Cardiology, vol. 55, no. 22, pp. 2470-2479, 2010.

[26] K. C. Wu, E. A. Zerhouni, R. M. Judd et al., "Prognostic significance of microvascular obstruction by magnetic resonance imaging in patients with acute myocardial infarction," Circulation, vol. 97, no. 8, pp. 765-772, 1998. 
[27] H. Yokota, S. Heidary, C. K. Katikireddy et al., "Quantitative characterization of myocardial infarction by cardiovascular magnetic resonance predicts future cardiovascular events in patients with ischemic cardiomyopathy," Journal of Cardiovascular Magnetic Resonance, vol. 10, no. 1, article 17, 2008.

[28] B. Y. C. Cheong, R. Muthupillai, J. M. Wilson et al., "Prognostic significance of delayed-enhancement magnetic resonance imaging: survival of 857 patients with and without left ventricular dysfunction," Circulation, vol. 120, no. 21, pp. 2069-2076, 2009.

[29] E. Larose, J. Rodés-Cabau, P. Pibarot et al., "Predicting late myocardial recovery and outcomes in the early hours of ST-segment elevation myocardial infarction. Traditional measures compared with microvascular obstruction, salvaged myocardium, and necrosis characteristics by cardiovascular magnetic resonance," Journal of the American College of Cardiology, vol. 55, no. 22, pp. 2459-2469, 2010.

[30] E. Wu, J. T. Ortiz, P. Tejedor et al., "Infarct size by contrast enhanced cardiac magnetic resonance is a stronger predictor of outcomes than left ventricular ejection fraction or endsystolic volume index: prospective cohort study," Heart, vol. 94, no. 6, pp. 730-736, 2008.

[31] T. Reffelmann and R. A. Kloner, "Microvascular reperfusion injury: tapid expansion of anatomic no reflow during reperfusion in the rabbit," The American Journal of Physiology, vol. 283, no. 3, pp. H1099-H1107, 2002.

[32] G. Ambrosio, H. F. Weisman, J. A. Mannisi, and L. C. Becker, "Progressive impairment of regional myocardial perfusion after initial restoration of postischemic blood flow," Circulation, vol. 80, no. 6, pp. 1846-1861, 1989.

[33] C. E. Rochitte, J. A. C. Lima, D. A. Bluemke et al., "Magnitude and time course of microvascular obstruction and tissue injury after acute myocardial infarction," Circulation, vol. 98, no. 10, pp. 1006-1014, 1998.

[34] K. C. Wu, R. J. Kim, D. A. Bluemke et al., "Quantification and time course of microvascular obstruction by contrastenhanced echocardiography and magnetic resonance imaging following acute myocardial infarction and reperfusion," Journal of the American College of Cardiology, vol. 32, no. 6, pp. 1756-1764, 1998.

[35] S. Orn, C. Manhenke, O. J. Greve et al., "Microvascular obstruction is a major determinant of infarct healing and subsequent left ventricular remodelling following primary percutaneous coronary intervention," European Heart Journal, vol. 30, no. 16, pp. 1978-1985, 2009.

[36] V. Hombach, O. Grebe, N. Merkle et al., "Sequelae of acute myocardial infarction regarding cardiac structure and function and their prognostic significance as assessed by magnetic resonance imaging," European Heart Journal, vol. 26, no. 6, pp. 549-557, 2005.

[37] O. Bruder, F. Breuckmann, C. Jensen et al., "Prognostic impact of contrast-enhanced CMR early after acute ST segment elevation myocardial infarction (STEMI) in a regional STEMI network," Herz, vol. 33, no. 2, pp. 136-142, 2008.

[38] R. Nijveldt, M. B. M. Hofman, A. Hirsch et al., "Assessment of microvascular obstruction and prediction of short-term remodeling after acute mycoardial infarction: cardiac MR imaging study," Radiology, vol. 250, no. 2, pp. 363-370, 2009.

[39] D. Garcia-Dorado, J. Oliveras, J. Gili et al., "Analysis of myocardial oedema by magnetic resonance imaging early after coronary artery occlusion with or without reperfusion," Cardiovascular Research, vol. 27, no. 8, pp. 1462-1469, 1993.
[40] A. H. Aletras, G. S. Tilak, A. Natanzon et al., "Retrospective determination of the area at risk for reperfused acute myocardial infarction with T2-weighted cardiac magnetic resonance imaging-histopathological and displacement encoding with stimulated echoes (DENSE) functional validations," Circulation, vol. 113, no. 15, pp. 1865-1870, 2006.

[41] M. Francone, C. Bucciarelli-Ducci, I. Carbone et al., "Impact of primary coronary angioplasty delay on myocardial salvage, infarct size, and microvascular damage in patients with ST-segment elevation myocardial infarction. Insight from cardiovascular magnetic resonance," Journal of the American College of Cardiology, vol. 54, no. 23, pp. 2145-2153, 2009.

[42] H. Bueno, R. LopezPalop, J. Bermejo, J. L. LopezSendon, and J. L. Delcan, "In-hospital outcome of elderly patients with acute inferior myocardial infarction and right ventricular involvement," Circulation, vol. 96, no. 2, pp. 436-441, 1997.

[43] M. Zehender, W. Kasper, E. Kauder et al., "Right ventricular infarction as an independent predictor of prognosis after acute inferior myocardial infarction," The New England Journal of Medicine, vol. 328, no. 14, pp. 981-988, 1993.

[44] A. Kumar, H. Abdel-Aty, I. Kriedemann et al., "Contrastenhanced cardiovascular magnetic resonance imaging of right ventricular infarction," Journal of the American College of Cardiology, vol. 48, no. 10, pp. 1969-1976, 2006.

[45] C. J. Jensen, M. Jochims, P. Hunold, G. V. Sabin, T. Schlosser, and O. Bruder, "Right ventricular involvement in acute left ventricular myocardial infarction: prognostic implications of MRI findings," The American Journal of Roentgenology, vol. 194, no. 3, pp. 592-598, 2010.

[46] T. A. M. Kaandorp, H. J. Lamb, D. Poldermans et al., "Assessment of right ventricular infarction with contrast-enhanced magnetic resonance imaging," Coronary Artery Disease, vol. 18, no. 1, pp. 39-43, 2007.

[47] C. M. Kramer, W. J. Rogers, G. Geskin et al., "Usefulness of magnetic resonance imaging early after acute myocardial infarction," The American Journal of Cardiology, vol. 80, no. 6, pp. 690-695, 1997.

[48] C. F. Azevedo, L. C. Amado, D. L. Kraitchman et al., "Persistent diastolic dysfunction despite complete systolic functional recovery after reperfused acute myocardial infarction demonstrated by tagged magnetic resonance imaging," European Heart Journal, vol. 25, no. 16, pp. 1419-1427, 2004.

[49] S. Okayama, S. Uemura, T. Soeda et al., "Clinical significance of papillary muscle late enhancement detected via cardiac magnetic resonance imaging in patients with single old myocardial infarction," International Journal of Cardiology, vol. 146, no. 1, pp. 73-79, 2010.

[50] A. M. Taylor, S. Dymarkowski, E. K. Verbeken, and J. Bogaert, "Detection of pericardial inflammation with lateenhancement cardiac magnetic resonance imaging: initial results," European Radiology, vol. 16, no. 3, pp. 569-574, 2006.

[51] M. B. Srichai, C. Junor, L. L. Rodriguez et al., "Clinical, imaging, and pathological characteristics of left ventricular thrombus: a comparison of contrast-enhanced magnetic resonance imaging, transthoracic echocardiography, and transesophageal echocardiography with surgical or pathological validation," The American Heart Journal, vol. 152, no. 1, pp. 75-84, 2006.

[52] J. W. Weinsaft, H. W. Kim, D. J. Shah et al., "Detection of left ventricular thrombus by delayed-enhancement cardiovascular magnetic resonance-prevalence and markers in patients with systolic dysfunction," Journal of the American College of Cardiology, vol. 52, no. 2, pp. 148-157, 2008. 
[53] S. C. Greaves, G. Zhi, R. T. Lee et al., "Incidence and natural history of left ventricular thrombus following anterior wall acute myocardial infarction," The American Journal of Cardiology, vol. 80, no. 4, pp. 442-448, 1997.

[54] F. Chiarella, E. Santoro, S. Domenicucci, A. Maggioni, and C. Vecchio, "Predischarge two-dimensional echocardiographic evaluation of left ventricular thrombosis after acute myocardial infarction in the GISSI-3 study," The American Journal of Cardiology, vol. 81, no. 7, pp. 822-827, 1998.

[55] J. W. Weinsaft, R. J. Kim, M. Ross et al., "Contrast-enhanced anatomic imaging as compared to contrast-enhanced tissue characterization for detection of left ventricular thrombus," JACC: Cardiovascular Imaging, vol. 2, no. 8, pp. 969-979, 2009.

[56] I. Meryon, N. Patel, T. Millane, and C. Varma, "Normal coronary angiography and primary percutaneous coronary intervention for ST elevation myocardial infarction: a literature review and audit findings," International Journal of Clinical Practice, vol. 64, no. 9, pp. 1245-1251, 2010.

[57] H. Mahrholdt, A. Wagner, C. C. Deluigi et al., "Presentation, patterns of myocardial damage, and clinical course of viral myocarditis," Circulation, vol. 114, no. 15, pp. 1581-1590, 2006.

[58] S. N. Yelgec, S. Dymarkowski, J. Ganame, and J. Bogaert, "Value of MRI in patients with a clinical suspicion of acute myocarditis," European Radiology, vol. 17, no. 9, pp. 22112217, 2007.

[59] D. M. Larson, K. M. Menssen, S. W. Sharkey et al., "Falsepositive' cardiac catheterization laboratory activation among patients with suspected ST-segment elevation myocardial infarction," Journal of the American Medical Association, vol. 298, no. 23, pp. 2754-2760, 2007.

[60] R. G. Assomull, J. C. Lyne, N. Keenan et al., "The role of cardiovascular magnetic resonance in patients presenting with chest pain, raised troponin, and unobstructed coronary arteries," European Heart Journal, vol. 28, no. 10, pp. 12421249, 2007.

[61] S. B. Joshi, T. Chao, D. A. Herzka et al., "Cardiovascular magnetic resonance T2 signal abnormalities in left ventricular ballooning syndrome," International Journal of Cardiovascular Imaging, vol. 26, no. 2, pp. 227-232, 2010.

[62] H. Abdel-Aty, M. Cocker, and M. G. Friedrich, "Myocardial edema is a feature of Tako-Tsubo cardiomyopathy and is related to the severity of systolic dysfunction: insights from T2-weighted cardiovascular magnetic resonance," International Journal of Cardiology, vol. 132, no. 2, pp. 291-293, 2009.

[63] H. Abdel-Aty, P. Boyé, A. Zagrosek et al., "Diagnostic performance of cardiovascular magnetic resonance in patients with suspected acute myocarditis: comparison of different approaches," Journal of the American College of Cardiology, vol. 45, no. 11, pp. 1815-1822, 2005.

[64] S. Nomura, N. Funabashi, M. Tsubura et al., "Cardiac sarcoidosis evaluated by multimodality imaging," International Journal of Cardiology, vol. 150, no. 2, pp. e81-e84, 2010.

[65] K. L. Davis, U. Mehlhorn, G. A. Laine, and S. J. Allen, "Myocardial edema, left ventricular function, and pulmonary hypertension," Journal of Applied Physiology, vol. 78, no. 1, pp. 132-137, 1995.

[66] C. R. Butler, R. Thompson, M. Haykowsky, M. Toma, and I. Paterson, "Cardiovascular magnetic resonance in the diagnosis of acute heart transplant rejection: a review," Journal of Cardiovascular Magnetic Resonance, vol. 11, no. 1, article 7, 2009. 


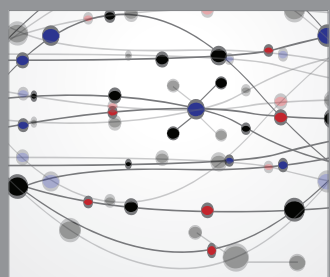

The Scientific World Journal
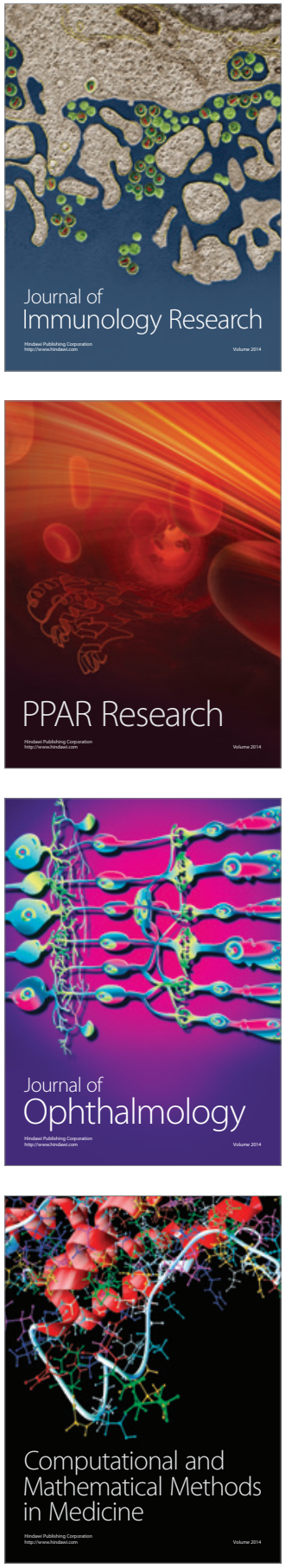

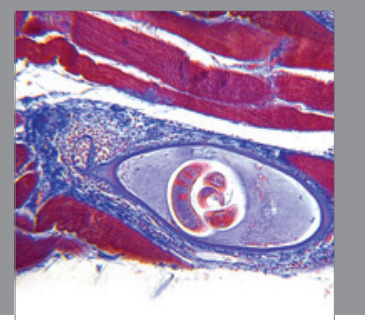

Gastroenterology

Research and Practice
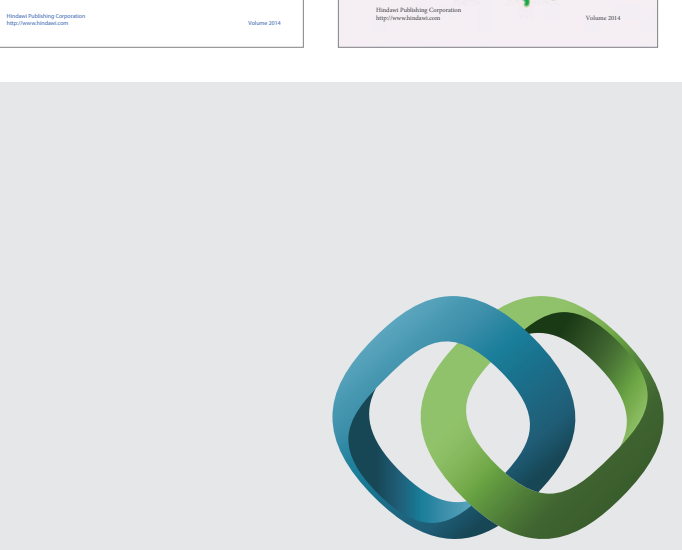

\section{Hindawi}

Submit your manuscripts at

http://www.hindawi.com
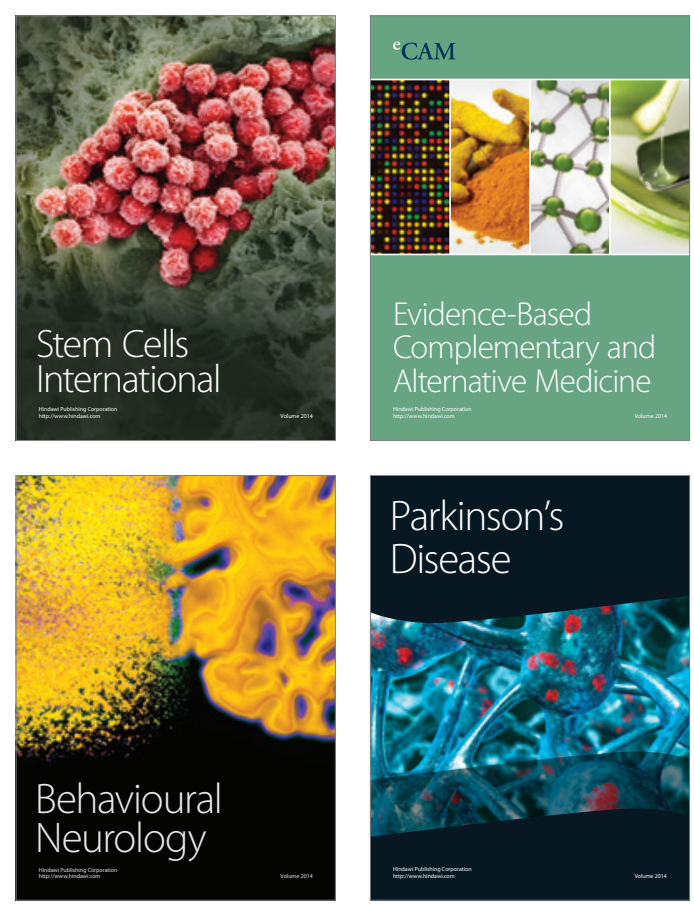

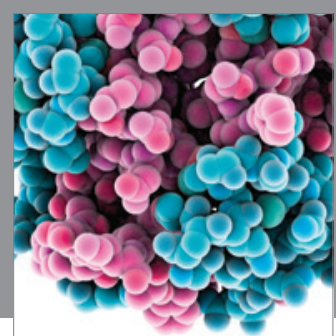

Journal of
Diabetes Research

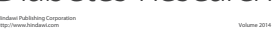

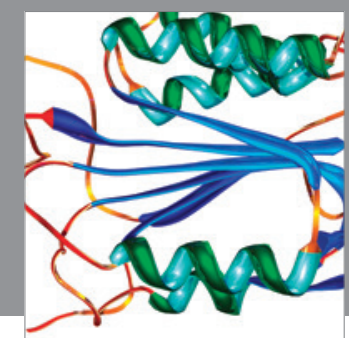

Disease Markers
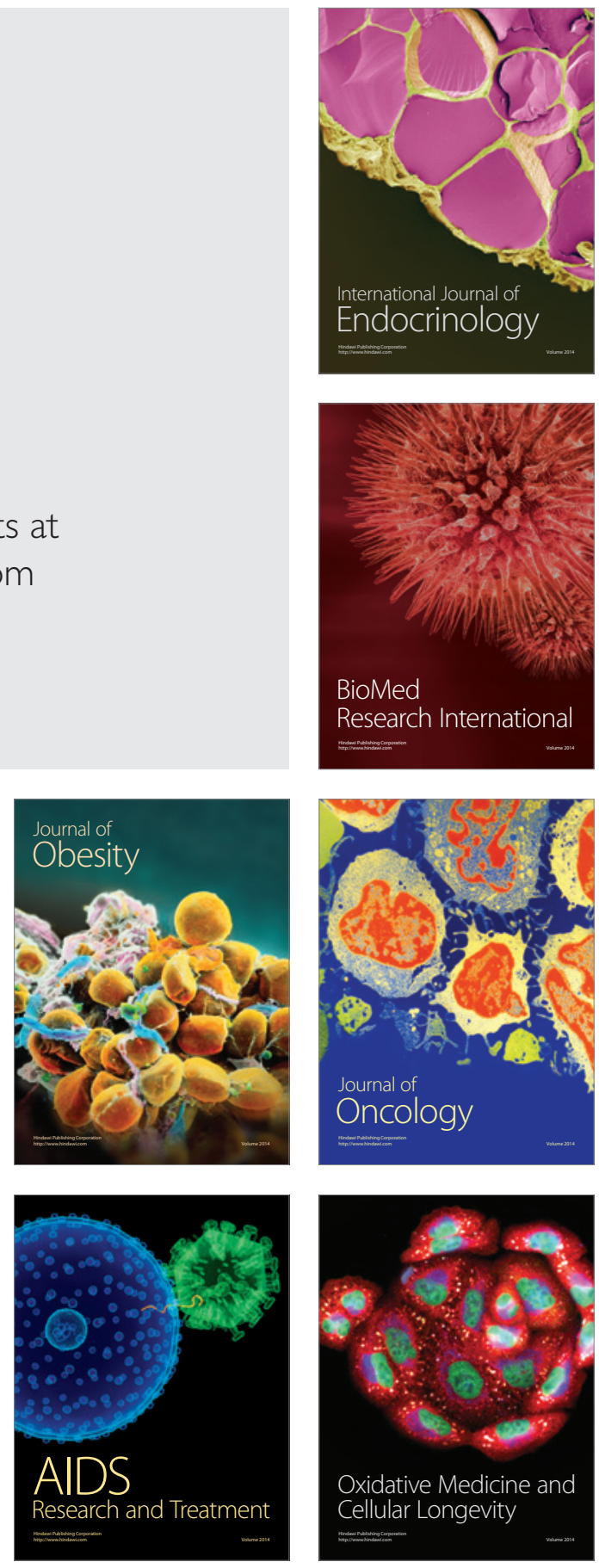